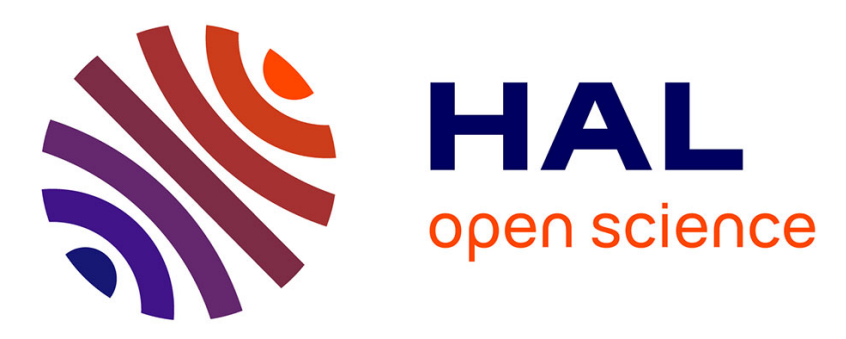

\title{
Numerical study of a thin liquid film flowing down an inclined wavy plane
}

Alexandre Ern, Rémi Joubaud, Tony Lelièvre

\section{To cite this version:}

Alexandre Ern, Rémi Joubaud, Tony Lelièvre. Numerical study of a thin liquid film flowing down an inclined wavy plane. Physica D: Nonlinear Phenomena, 2011, 240 (21), pp.1714-1723. 10.1016/j.physd.2011.07.007 . hal-00533000v2

\section{HAL Id: hal-00533000 https://hal.science/hal-00533000v2}

Submitted on 2 Apr 2012

HAL is a multi-disciplinary open access archive for the deposit and dissemination of scientific research documents, whether they are published or not. The documents may come from teaching and research institutions in France or abroad, or from public or private research centers.
L'archive ouverte pluridisciplinaire HAL, est destinée au dépôt et à la diffusion de documents scientifiques de niveau recherche, publiés ou non, émanant des établissements d'enseignement et de recherche français ou étrangers, des laboratoires publics ou privés. 


\title{
Numerical study of a thin liquid film flowing down an inclined wavy plane
}

\author{
Alexandre Ern $^{\mathrm{a}}$, Rémi Joubaud ${ }^{\mathrm{a}}$, Tony Lelièvre ${ }^{\mathrm{a}}$ \\ ${ }^{a}$ Université Paris-Est, CERMICS, Ecole des Ponts, 6 \&3 8 Av. B. Pascal, 77455 \\ Marne-la-Vallée cedex 2, France
}

\begin{abstract}
We investigate the stability of a thin liquid film flowing down an inclined wavy plane using a direct numerical solver based on a finite element/arbitrary Lagrangian Eulerian approximation of the free-surface Navier-Stokes equations. We study the dependence of the critical Reynolds number for the onset of surface wave instabilities on the inclination angle, the waviness parameter, and the wavelength parameter, focusing in particular on mild inclinations and relatively large waviness so that the bottom does not fall monotonously. In the present parameter range, shorter wavelengths and higher amplitude for the bottom undulation stabilize the flow. The dependence of the critical Reynolds number evaluated with the Nusselt flow rate on the inclination angle is more complex than the classical relation (5/6 times the cotangent of the inclination angle), but this dependence can be recovered if the actual flow rate at critical conditions is used instead.
\end{abstract}

Key words: free-surface Navier-Stokes, thin liquid film, surface wave instability, critical Reynolds number, direct numerical simulation, ALE formulation PACS: 02.60.Cb, 47.10.ad, 47.15.gm

2000 MSC: 65P40, 76D05, 76E99, 76M99

\section{Introduction}

The original motivation for this work is the derivation of hydrological models to predict overland flows within small agricultural watersheds where the flow direction is not only controlled by the topography but also, within the fields, by the presence of ridges and furrows created by tillage operations [11]. Such a study is currently being carried over by soil engineers together with applied mathematicians within the project METHODE [7]. 
We investigate here more specifically instabilities that can occur when a thin gravity-driven film flows down an inclined wavy plane representing the furrows within a cultivated field. Film flow along wavy walls has other interesting engineering applications, for instance in two-phase heat exchangers [12]. However, in the present work, we mainly focus on mildly inclined planes as encountered in agricultural watersheds.

Thin gravity-driven films flowing down an inclined flat plane provide one of the simplest configurations where hydrodynamics instabilities can occur, even at relatively low flow rates. This setting exhibits a rich phenomenology and, as such, has prompted a substantial amount of theoretical, experimental, and numerical work over the past decades, aiming at predicting the onset of instability and also at analyzing the development and possible disorganization of the waves at the surface of the liquid film. The first theoretical description of the flow down a perfectly flat incline can be traced back to the seminal work of Nusselt who studied film condensation on vertical walls [8]. A particular stationary solution of the free-surface Navier-Stokes equations is indeed the so-called Nusselt flow which is a boundary layer type flow featuring constant height, parabolic velocity profile, while the flow rate is determined by balancing the work of gravity with viscous dissipation. Further understanding of surface wave instabilities was achieved in the works of Benjamin [1] and Yih [15], still in the case of a perfectly flat inclined plane. One of the main results was the condition for the flat Nusselt solution to be unstable against long wavelength infinitesimal perturbations (that is, wavelengths that are large compared to the thickness of the film) in terms of a critical Reynolds number $\operatorname{Re}_{c}=(5 / 6) \cot \theta$. Here, $\theta$ denotes the angle of the inclined plane with the horizontal line, while the constant $5 / 6$ depends on the definition for the Reynolds number, the present value being obtained using the film thickness as reference length and the Nusselt velocity as reference velocity. Concerning more recent work, without being exhaustive, let us mention the experimental work of Liu and Gollub [6] and the numerical work of Ramaswamy, Chippada and Joo [9] investigating the transition from nearly sinusoidal permanent waveforms to solitary humps as well as complex wave processes such as wave merging and wave splitting. We also mention the work of Ruyer-Quil and Manneville [10] who proposed a new class of models (so-called higher-order shallow-water models) to formulate the free-surface flow problem and obtained results in good agreement with both experiments and direct numerical simulations.

In contrast to the above literature dedicated to flows over inclined flat planes, much less studies are available in the case of flows over inclined wavy planes. Important results in this direction have been achieved by Wierschem, 
Aksel, and Scholle $[13,14]$ using an analytical approach, similar in spirit to that of Yih [15] for a flat plane, based on an expansion of the free-surface Navier-Stokes equations in which the wavelength parameter (essentially the ratio of the film thickness to the wavelength of the bottom undulations) serves as the perturbation parameter. The main result is that the critical Reynolds number for the onset of surface waves is higher than that for a flat bottom. In other words, the waviness in the topography has a stabilizing effect on the flow (although, under certain conditions, the film flow can be destabilized locally at steeper slopes). Analytical formulas for the film thickness, velocities and pressure profiles are also derived. However, one limitation of the above analysis is the requirement of monotonously falling bottom contours, that is, for a given inclination angle, the waviness of the topography cannot be too large. This prevents the application of the above results to the setting of interest here where the agricultural field exhibits a mild inclination while the furrows induce a sufficiently large waviness so that the bottom contour can raise locally.

The present work's principal aim is to fill this gap using direct numerical simulations of the free-surface Navier-Stokes equations. For completeness, a brief comparison with a shallow-water model is also discussed. As in [13], the deviation from the flat topography is modeled using a sinusoidal perturbation. Since close to the instability threshold, surface waves are essentially streamwise surface undulations free of spanwise modulations [10], the numerical simulations are performed in a two-dimensional setting. Moreover, the flow domain stretches over one wavelength of the sinusoidal perturbation of the topography, and along its lateral sides, periodic boundary conditions are enforced. Within this domain, the free-surface Navier-Stokes equations are solved numerically using finite elements for space discretization and an Arbitrary Lagrangian Eulerian (ALE) method to track the free surface. We are especially interested in studying the critical Reynolds number for the onset of surface wave instabilities as a function of the mean inclination angle of the topography, the waviness parameter measuring the amplitude of the deviation from the flat topography, and the wavelength parameter mentioned above. Herein, we do not consider surface tension effects, that is, we assume that the capillary length is smaller than the wavelength of the bottom undulations. We refer to [13] for a study including surface tension, where it is found that owing to its stabilizing effect, the latter can slightly alter the critical Reynolds number.

To determine the critical Reynolds number, we proceed as follows. Two stable configurations are simulated first by selecting two values for the viscosity that are large enough. In both cases, we observe that perturbations 
decay exponentially in time at the free surface; the critical value for the Reynolds number is then obtained by extrapolation on the viscosity in such a way that the extrapolated decay rate vanishes. To evaluate the Reynolds number, a reference length and a reference velocity must be specified. For the former, it is natural to use the mean film thickness based on volume conservation. For the latter, we observe that the flow rate at steady-state is not known a priori. As for the Nusselt flow over an inclined flat plane, the flow rate results from the balance between the work of gravity and viscous dissipation, but an analytical calculation of the viscous dissipation is no longer possible for wavy planes because the flow profile is no longer parabolic and it depends on the streamwise coordinate. A quite reasonable choice, as in [13], is to use as reference velocity the Nusselt flow velocity over a flat plane with the same inclination angle. However, for relatively high amplitudes of the bottom undulations, the actual flow rate at steady-state can differ from the Nusselt flow rate by up to $25 \%$. It is therefore interesting to discuss our results also by using the actual flow rate to evaluate the critical Reynolds number. Since our results are inferred from computations at fixed positive values for the wavelength parameter, we verify our numerical protocol to compute the critical Reynolds number by comparing our results to those of [13] for small enough wavelength parameter and small enough waviness parameter for the bottom to fall monotonously.

One interesting result presented hereafter is that the ratio of critical Reynolds number (with Nusselt flow velocity) to $\cot \theta$ still mildly depends on $\theta$, while this dependence almost disappears if the actual flow rate is used. Moreover, long wavelength disturbances are more prone to destabilize the flow. Higher amplitudes for the bottom undulations lead to larger values for the critical Reynolds number evaluated with the Nusselt flow velocity, while the Reynolds number evaluated with the actual flow rate is almost independent of the waviness parameter. Finally, the free surface obtained by the Navier-Stokes simulations can be fairly well reproduced by a shallowwater model even for relatively large values of the wavelength parameter.

The rest of this work is organized as follows. In Section 2 we present the physical setting in more detail. In Section 3 we briefly recall the main features of the finite element/ALE discretization of the free-surface NavierStokes equations. Finally, in Section 4 we describe the numerical protocol to determine the critical Reynolds number and discuss our results. 


\section{Physical setting}

In this section, we describe the geometric set-up for solving the freesurface Navier-Stokes equations. We also briefly recall the Nusselt flow solution over an inclined flat plane and specify the time and length scales to formulate the governing equations in non-dimensional form.

\subsection{Free-surface Navier-Stokes equations}

Referring to Figure 1, we consider the movement of a two-dimensional thin film flowing down a rigid surface of finite length $L$ periodized with $L$ periodicity. This surface consists of a plane inclined with angle $0 \leq \theta \leq \pi / 2$ and perturbed sinusoidally. The Cartesian coordinates are denoted by $\boldsymbol{x}=$ $\left(x_{1}, x_{2}\right)$ where $x_{1}$ follows the inclined plane. By periodicity, $x_{1}$ belongs to the torus $\mathbb{T}=\mathbb{R} / L \mathbb{Z}$. With the sinusoidal perturbation, the elevation of the topography along the $x_{2}$-axis is given by

$$
b\left(x_{1}\right)=A \sin \left(\frac{2 \pi x_{1}}{L}\right), \quad x_{1} \in \mathbb{T} .
$$

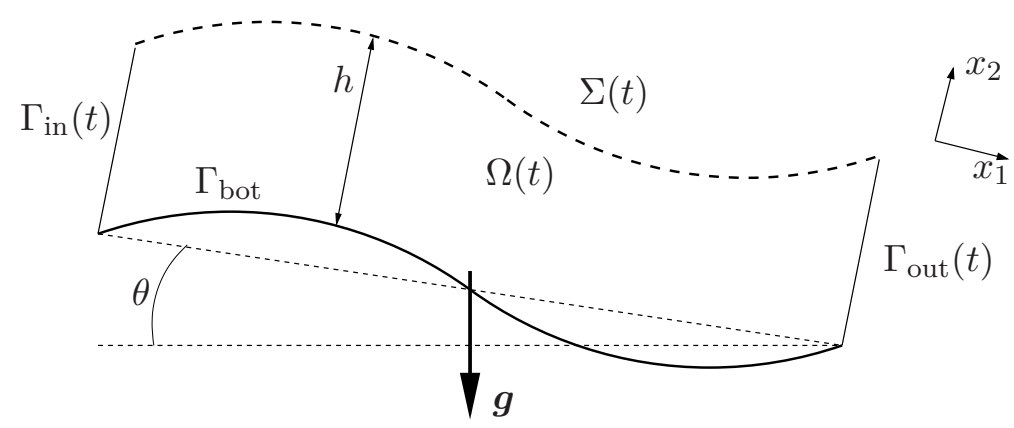

Figure 1: Geometric set-up.

At any time $t \geq 0$, the fluid occupies a domain denoted by $\Omega(t)$. The boundary $\partial \Omega(t)$ of $\Omega(t)$ is partitioned as follows (see Figure 1):

$$
\begin{aligned}
\Sigma(t) & =\left\{\boldsymbol{x} \in \mathbb{T} \times \mathbb{R}, x_{2}=h\left(t, x_{1}\right)\right\}, \\
\Gamma_{\text {bot }} & =\left\{\boldsymbol{x} \in \mathbb{T} \times \mathbb{R}, x_{2}=b\left(x_{1}\right)\right\}, \\
\Gamma_{\text {in/out }}(t) & =\left\{\boldsymbol{x} \in \mathbb{T} \times \mathbb{R}, x_{1} \in\{0, L\}, 0 \leq x_{2} \leq h(t, 0)\right\} .
\end{aligned}
$$

Here, $\Sigma(t)$ is the air/liquid interface to which we will refer as the free surface, $h$ is the fluid thickness evolving with time (by periodicity, $h(t, 0)=h(t, L)$ ), $\Gamma_{\text {bot }}$ is the rigid bottom (with $b$ defined by $(1)$ ), and $\Gamma_{\text {in/out }}(t)$ are the lateral 
boundaries associated with periodicity. We observe that only $\Gamma_{\text {bot }}$ is timeindependent. In what follows, $\boldsymbol{n}$ denotes the unit outward vector normal to $\partial \Omega$. In view of the ALE framework to be considered later, it is convenient to relate the current frame $\Omega(t)$ to a reference frame $\hat{\Omega}$. To this purpose, we assume that for any time $t \geq 0$, there exists a smooth and bijective map $\hat{\mathcal{A}}_{t}$ from a reference domain $\hat{\Omega}$ to the current domain $\Omega(t)$ such that $\hat{\mathcal{A}}_{t}(\hat{\Omega})=\Omega(t)$. The inverse map (with respect to the space variable) of $\hat{\mathcal{A}}_{t}$ is denoted $\hat{\mathcal{A}}_{t}^{-1}$. The velocity of the domain $\hat{\boldsymbol{w}}$ is defined as

$$
\hat{\boldsymbol{w}}(t, \hat{\boldsymbol{x}})=\frac{\partial}{\partial t} \hat{\mathcal{A}}_{t}(\hat{\boldsymbol{x}})
$$

To any function $\psi(t, \cdot)$ defined on the current frame $\Omega(t)$ is associated the function $\hat{\psi}(t, \cdot)$ defined on the reference domain $\hat{\Omega}$ by $\hat{\psi}(t, \hat{\boldsymbol{x}})=\psi\left(t, \hat{\mathcal{A}}_{t}(\hat{\boldsymbol{x}})\right)$. For example, the velocity of the domain $\boldsymbol{w}$ on the current frame is defined as

$$
\boldsymbol{w}(t, \boldsymbol{x})=\hat{\boldsymbol{w}}\left(t, \hat{\mathcal{A}}_{t}^{-1}(\boldsymbol{x})\right) .
$$

By periodicity,

$$
\left.\boldsymbol{w}(t, \cdot)\right|_{\Gamma_{\text {in }}(t)}=\left.\boldsymbol{w}(t, \cdot)\right|_{\Gamma_{\text {out }}(t)},
$$

with $\left.\boldsymbol{w}(t, \cdot) \cdot \boldsymbol{n}\right|_{\Gamma_{\text {in } / \text { out }}(t)}=0$, and since the bottom represents a rigid surface,

$$
\left.\boldsymbol{w}(t, \cdot)\right|_{\Gamma_{\text {bot }}}=\mathbf{0}
$$

We assume the fluid to be Newtonian, isothermal, and incompressible. Its motion is governed by the Navier-Stokes equations which express the conservation of momentum and mass in the form

$$
\left\{\begin{array}{l}
\partial_{t}(\rho \boldsymbol{u})+\operatorname{div}(\rho \boldsymbol{u} \otimes \boldsymbol{u})-\operatorname{div} \boldsymbol{\sigma}(\boldsymbol{u}, p)=\rho \boldsymbol{g}, \\
\operatorname{div}(\boldsymbol{u})=0
\end{array}\right.
$$

Here, $\boldsymbol{u}$ is the fluid velocity with Cartesian components $\left(u_{1}, u_{2}\right), \rho$ the density, $\boldsymbol{\sigma}(\boldsymbol{u}, p)$ the stress tensor given by

$$
\boldsymbol{\sigma}(\boldsymbol{u}, p)=-p \operatorname{Id}+2 \eta \mathcal{E}(\boldsymbol{u}),
$$

where $p$ is the pressure, $\eta$ the (dynamic) viscosity, $\mathcal{E}(\boldsymbol{u})=\frac{1}{2}\left(\nabla \boldsymbol{u}+\nabla \boldsymbol{u}^{T}\right)$ the linearized strain tensor, and finally, the gravity forces are given by

$$
\boldsymbol{g}=g \boldsymbol{\Theta}=\left(\begin{array}{c}
g \sin \theta \\
-g \cos \theta
\end{array}\right),
$$


with $g$ the constant of gravity acceleration. The Navier-Stokes equations (6) are complemented with initial conditions specifying $\boldsymbol{u}(t=0)$ and $\Omega(t=0)$ and with boundary conditions. The latter enforce no-penetration and no-slip conditions at the bottom

$$
\boldsymbol{u}=\mathbf{0} \text { on } \Gamma_{\text {bot }},
$$

zero stress at the free surface (thereby neglecting surface tension)

$$
\boldsymbol{\sigma}(\boldsymbol{u}, p) \boldsymbol{n}=\mathbf{0} \text { on } \Sigma(t),
$$

and periodicity for velocity and the normal component of $\boldsymbol{\sigma}(\boldsymbol{u}, p)$ on $\Gamma_{\text {in/out }}(t)$. Finally, the fact that the free surface $\Sigma(t)$ is a material line is expressed by the kinematic condition

$$
\boldsymbol{w} \cdot \boldsymbol{n}=\boldsymbol{u} \cdot \boldsymbol{n} \text { on } \Sigma(t) .
$$

There are several possibilities to define the domain velocity $\boldsymbol{w}$ inside $\Omega(t)$ matching the boundary conditions (4), (5), and (11). A simple choice based on solving a scalar Poisson problem is presented in Section 3.

\subsection{Volume and energy conservation}

Two classical properties of the free-surface Navier-Stokes equations are volume and energy conservation. The former can simply be expressed as

$$
\frac{d}{d t}|\Omega(t)|=0
$$

Moreover, the energy balance takes the form

$$
\frac{d}{d t} K(t)+P_{v}(t)=\int_{\Omega(t)} \rho \boldsymbol{g} \cdot \boldsymbol{u} d \boldsymbol{x},
$$

where $K(t)$ denotes the kinetic energy of the fluid at time $t$ given by

$$
K(t)=\frac{1}{2} \int_{\Omega(t)} \rho|\boldsymbol{u}|^{2} d \boldsymbol{x},
$$

and $P_{v}(t)$ the viscous dissipation at time $t$ given by

$$
P_{v}(t)=\int_{\Omega(t)} \frac{\eta}{2}\left|\nabla \boldsymbol{u}+\nabla \boldsymbol{u}^{T}\right|^{2} d \boldsymbol{x} .
$$

For completeness, we briefly recall the derivation of these two properties [4]. In the present ALE framework, the Reynolds transport formula states that for any smooth function $\varphi$ depending on time $t$ and space $\boldsymbol{x}$,

$$
\frac{d}{d t} \int_{\Omega(t)} \varphi d \boldsymbol{x}=\int_{\Omega(t)} \partial_{t} \varphi d \boldsymbol{x}+\int_{\partial \Omega(t)} \varphi \boldsymbol{w} \cdot \boldsymbol{n} d \sigma
$$


and accounting for periodicity and rigid bottom yields

$$
\frac{d}{d t} \int_{\Omega(t)} \varphi d \boldsymbol{x}=\int_{\Omega(t)} \partial_{t} \varphi d \boldsymbol{x}+\int_{\Sigma(t)} \varphi \boldsymbol{w} \cdot \boldsymbol{n} d \sigma .
$$

Taking $\varphi \equiv 1$, using the kinematic condition (11) together with incompressibility and periodicity yields (12). Turning to energy balance, we multiply the momentum conservation equation in (6) by $\boldsymbol{u}$ and integrate over $\Omega(t)$ to infer

$$
\begin{array}{r}
\int_{\Omega(t)} \partial_{t}(\rho \boldsymbol{u}) \cdot \boldsymbol{u} d \boldsymbol{x}+\int_{\Omega(t)} \operatorname{div}(\rho \boldsymbol{u} \otimes \boldsymbol{u}) \cdot \boldsymbol{u} d \boldsymbol{x}-\int_{\Omega(t)} \operatorname{div}(\boldsymbol{\sigma}(\boldsymbol{u}, p)) \cdot \boldsymbol{u} d \boldsymbol{x} \\
=\int_{\Omega(t)} \rho \boldsymbol{g} \cdot \boldsymbol{u} d \boldsymbol{x} .
\end{array}
$$

For the first term, we use the Reynolds transport formula with $\varphi \equiv \frac{1}{2} \rho|\boldsymbol{u}|^{2}$ yielding

$$
\int_{\Omega(t)} \partial_{t}(\rho \boldsymbol{u}) \cdot \boldsymbol{u} d \boldsymbol{x}=\frac{d}{d t} \int_{\Omega(t)} \frac{1}{2} \rho|\boldsymbol{u}|^{2} d \boldsymbol{x}-\int_{\Sigma(t)} \frac{1}{2} \rho|\boldsymbol{u}|^{2} \boldsymbol{w} \cdot \boldsymbol{n} d \sigma .
$$

For the second term, we integrate by parts and use incompressibility to obtain

$$
\int_{\Omega(t)} \operatorname{div}(\rho \boldsymbol{u} \otimes \boldsymbol{u}) \cdot \boldsymbol{u} d \boldsymbol{x}=\int_{\Sigma(t)} \frac{1}{2} \rho|\boldsymbol{u}|^{2} \boldsymbol{u} \cdot \boldsymbol{n} d \sigma,
$$

and proceeding similarly for the third term yields

$$
-\int_{\Omega(t)} \operatorname{div}(\boldsymbol{\sigma}(\boldsymbol{u}, p)) \cdot \boldsymbol{u} d \boldsymbol{x}=\int_{\Omega(t)} \frac{\eta}{2}\left|\nabla \boldsymbol{u}+\nabla \boldsymbol{u}^{T}\right|^{2} d \boldsymbol{x}-\int_{\partial \Omega(t)} \boldsymbol{\sigma}(\boldsymbol{u}, p) \boldsymbol{n} \cdot \boldsymbol{u} d \sigma
$$

and the second term on the right-hand side of (21) vanishes owing to the boundary conditions. Collecting these expressions and using the kinematic condition (11) yields the energy balance (13).

\subsection{Nusselt flow}

In the case of a perfectly flat inclined plane, that is, $A=0$ in (1), the freesurface Navier-Stokes equations with the above boundary conditions admit a well-known stationary solution, referred to as the Nusselt flow, for which the film thickness is constant and the velocity profile is parabolic. Denoting by $h_{N}$ the film thickness, the velocity takes the form $\boldsymbol{u}(\boldsymbol{x})=\varphi\left(x_{2}\right) \boldsymbol{e}_{1}$ where 
$\boldsymbol{e}_{1}$ denotes the Cartesian basis vector associated with the first coordinate. The function $\varphi$ which determines the vertical velocity profile is given by

$$
\varphi\left(x_{2}\right)=\frac{3}{2} \frac{Q_{N}}{h_{N}}\left(\frac{2 x_{2}}{h_{N}}-\left(\frac{x_{2}}{h_{N}}\right)^{2}\right), \quad x_{2} \in\left[0, h_{N}\right],
$$

where $Q_{N}=\int_{0}^{h_{N}} \varphi\left(x_{2}\right) d x_{2}$ is the flow rate so that $Q_{N} / h_{N}$ is the mean flow velocity. The flow rate results from the energy balance (13) at steady-state, and a straightforward computation yields

$$
Q_{N}=\frac{1}{3} \frac{\rho g \sin \theta}{\eta} h_{N}^{3}
$$

\subsection{Scaling and non-dimensionalization}

In the case of an inclined wavy plane, an analytic expression of the steady-state solution of the free-surface Navier-Stokes equations (if such a solution exists) is no longer available. In particular, the film thickness is no longer constant, and the velocity profile is no longer parabolic. To formulate the equations in non-dimensional form, we use as reference length the mean film thickness in space. Owing to the volume conservation property (12), this reference length does not depend on time and can be determined from the initial fluid domain $\Omega_{0}$. In what follows, we denote this reference length by $h_{*}$. Furthermore, a reasonable choice for the reference velocity, say $U_{*}$, is the mean flow velocity corresponding to the Nusselt flow with film thickness $h_{*}$, so that using (23) we obtain

$$
U_{*}=\frac{1}{3} \frac{\rho g \sin \theta}{\eta} h_{*}^{2} .
$$

Finally, we classically consider the advective time scale $t_{*}=h_{*} / U_{*}$ for the reference time and the Bernoulli scaling $\rho U_{*}^{2}$ for the pressure. With the above choices for the various scales, the free-surface Navier-Stokes equations (6) can be rewritten in the following non-dimensional form (for simplicity, we use the same notation for non-dimensional quantities):

$$
\left\{\begin{array}{l}
\partial_{t} \boldsymbol{u}+\operatorname{div}(\boldsymbol{u} \otimes \boldsymbol{u})-\operatorname{div}\left(\frac{2}{\operatorname{Re}} \mathcal{E}(\boldsymbol{u})\right)+\nabla p=\frac{1}{\mathrm{Fr}^{2}} \boldsymbol{\Theta} \\
\operatorname{div}(\boldsymbol{u})=0
\end{array}\right.
$$

with the Reynolds number defined by

$$
\operatorname{Re}:=\frac{\rho U_{*} h_{*}}{\eta}=\frac{1}{3}(\sin \theta) \frac{\rho^{2} g h_{*}^{3}}{\eta^{2}},
$$


while the Froude number is such that

$$
\operatorname{Fr}^{2}:=\frac{U_{*}^{2}}{h_{*} g}=\frac{1}{3}(\sin \theta) \operatorname{Re},
$$

owing to the scaling chosen for the velocity.

Henceforth, we are particularly interested in the critical value of the Reynolds number for the onset of surface wave instabilities. We want to investigate the dependence of this critical Reynolds number on the inclination angle $\theta$ and on two additional geometric parameters related to the topography, namely the waviness parameter $\zeta$ and the wavelength parameter $\xi$ defined by

$$
\zeta:=2 \pi \frac{A}{L}, \quad \xi:=2 \pi \frac{h_{*}}{L} .
$$

The parameter $\xi$ is also sometimes referred to as the thin-film parameter.

\section{Finite element/ALE solver}

We now briefly describe the numerical method used to solve the freesurface Navier-Stokes equations. More details can be found in $[3,4,5]$.

\subsection{Weak ALE formulation}

The weak ALE formulation is derived using test functions that do not depend on time in the reference frame $\hat{\Omega}$ whereas they do on the current frame $\Omega(t)$. More precisely, letting $T$ be the simulation time, for all $t \in$ $(0, T)$, the test spaces for velocity and pressure are defined using functions defined on the reference domain $\hat{\Omega}$ as follows:

$$
\begin{aligned}
V_{t} & =\left\{\boldsymbol{v}: \Omega(t) \rightarrow \mathbb{R}^{2} ; \boldsymbol{v}(t, \boldsymbol{x})=\hat{\boldsymbol{v}}\left(\hat{\mathcal{A}}_{t}^{-1}(\boldsymbol{x})\right) ; \hat{\boldsymbol{v}} \in \hat{V}\right\}, \\
M_{t} & =\left\{q: \Omega(t) \rightarrow \mathbb{R} ; q(t, \boldsymbol{x})=\hat{q}\left(\hat{\mathcal{A}}_{t}^{-1}(\boldsymbol{x})\right) ; \hat{q} \in \hat{M}\right\},
\end{aligned}
$$

where $\hat{V}:=\left\{\hat{\boldsymbol{v}} \in H^{1}(\hat{\Omega})^{2} ;\left.\hat{\boldsymbol{v}}\right|_{\Gamma_{\text {bot }}}=\mathbf{0} ; \hat{\boldsymbol{v}}\right.$ periodic $\}$ and $\hat{M}:=L^{2}(\hat{\Omega})$. Then, for all $t \in[0, T]$, we look for a map $\hat{\mathcal{A}}_{t}: \hat{\Omega} \rightarrow \Omega(t)$ and for functions $(\boldsymbol{u}(t), p(t))$ such that $(\boldsymbol{u}(t), p(t)) \in V_{t} \times M_{t}$ with $\int_{0}^{T} \int_{\Omega(t)}\left(p^{2}+|\nabla \boldsymbol{u}|^{2}\right) d \boldsymbol{x} d t<$ $+\infty$ and for all $(\boldsymbol{v}, q)$ in $V_{t} \times M_{t}$,

$$
\left\{\begin{array}{l}
\frac{d}{d t} \int_{\Omega(t)} \boldsymbol{u} \cdot \boldsymbol{v} d \boldsymbol{x}+\int_{\Omega(t)}(\boldsymbol{u}-\boldsymbol{w}) \cdot \nabla \boldsymbol{u} \cdot \boldsymbol{v} d \boldsymbol{x}-\int_{\Omega(t)} \operatorname{div}(\boldsymbol{w}) \boldsymbol{u} \cdot \boldsymbol{v} d \boldsymbol{x} \\
\quad+\int_{\Omega(t)} \frac{2}{\operatorname{Re}} \mathcal{E}(\boldsymbol{u}): \mathcal{E}(\boldsymbol{v}) d \boldsymbol{x}-\int_{\Omega(t)} p \operatorname{div}(\boldsymbol{v}) d \boldsymbol{x}=\int_{\Omega(t)} \frac{1}{\mathrm{Fr}^{2}} \boldsymbol{\Theta} \cdot \boldsymbol{v} d \boldsymbol{x} \\
\int_{\Omega(t)} q \operatorname{div}(\boldsymbol{u}) d \boldsymbol{x}=0
\end{array}\right.
$$


together with the initial conditions $\hat{\mathcal{A}}_{0}(\hat{\Omega})=\Omega_{0}$ and $\boldsymbol{u}(t=0, \cdot)=\boldsymbol{u}_{0}$. We recall that the domain velocity $\boldsymbol{w}$ satisfies the boundary conditions (4), (5), and (11).

\subsection{Time and space discretization}

The discretization is based on finite elements in space and a semi-implicit Euler scheme in time. Let $\delta t$ be the time step, taken constant for simplicity. We denote by $t^{n}=n \delta t$ the $n$-th discrete time. Given the velocity $\boldsymbol{u}^{n}$ discretized with finite elements at time $t^{n}$, we determine the mesh velocity $\boldsymbol{w}^{n}$ as described below by (36). Then, we introduce the map

$$
\mathcal{A}_{n, n+1}: \Omega^{n} \ni \boldsymbol{y} \longmapsto \boldsymbol{x}=\boldsymbol{y}+\delta t \boldsymbol{w}^{n}(\boldsymbol{y}) \in \Omega^{n+1},
$$

which can be seen as an approximation of the map $\hat{\mathcal{A}}_{t^{n+1}} \circ \hat{\mathcal{A}}_{t^{n}}^{-1}$. Given the spatial mesh at the discrete time $t^{n}$, say $\mathcal{M}^{n}$, the map $\mathcal{A}_{n, n+1}$ allows one to define the mesh at the discrete time $t^{n+1}$, say $\mathcal{M}^{n+1}$, by transporting each node of $\mathcal{M}^{n}$ from $\Omega^{n}$ to $\Omega^{n+1}$.

For space discretization, we consider at each discrete time $t^{n}$ mixed finite element spaces spanned by functions defined on $\Omega^{n}$ to approximate velocity and pressure, say $V_{h}^{n}$ and $M_{h}^{n}$. We strongly impose the essential velocity boundary condition on the bottom and enforce periodicity of the velocities by eliminating the corresponding degrees of freedom. We consider the $\mathbb{Q}_{2} / \mathbb{Q}_{1}$ setting, that is, continuous piecewise biquadratic finite elements for the velocity and continuous piecewise bilinear finite elements for the pressure. Using discontinuous piecewise affine finite elements for the pressure is also possible. The present choice yields faster convergence rates for the linear solver. Moreover, consistently with the weak ALE framework, the test functions follow the displacement of the domain given by $\mathcal{A}_{n, n+1}$, so that the test functions at the discrete time $t^{n+1}$ are in

$$
\begin{aligned}
V_{h}^{n+1} & =\left\{\boldsymbol{v}: \Omega^{n+1} \rightarrow \mathbb{R}^{2} ; \boldsymbol{v}(\boldsymbol{x})=\boldsymbol{v}\left(\mathcal{A}_{n, n+1}^{-1}(\boldsymbol{x})\right) ; \boldsymbol{v} \in V_{h}^{n}\right\}, \\
M_{h}^{n+1} & =\left\{q: \Omega^{n+1} \rightarrow \mathbb{R} ; q(\boldsymbol{x})=q\left(\mathcal{A}_{n, n+1}^{-1}(\boldsymbol{x})\right) ; q \in M_{h}^{n}\right\} .
\end{aligned}
$$

Finally, we discretize (31) in time with a semi-implicit Euler scheme. Thus, given $\boldsymbol{u}^{n} \in V_{h}^{n}, \Omega^{n}, \boldsymbol{w}^{n}$, and $\Omega^{n+1}$, we seek for $\left(\boldsymbol{u}^{n+1}, p^{n+1}\right) \in V_{h}^{n+1} \times M_{h}^{n+1}$ 
such that for all $(\boldsymbol{v}, q) \in V_{h}^{n+1} \times M_{h}^{n+1}$,

$$
\left\{\begin{array}{c}
\frac{1}{\delta t} \int_{\Omega^{n+1}} \boldsymbol{u}^{n+1} \cdot \boldsymbol{v} d \boldsymbol{x}+\int_{\Omega^{n+1}}\left(\tilde{\boldsymbol{u}}^{n}-\tilde{\boldsymbol{w}}^{n}\right) \cdot \nabla \boldsymbol{u}^{n+1} \cdot \boldsymbol{v} d \boldsymbol{x} \\
\quad-\int_{\Omega^{n+1}} \operatorname{div}\left(\tilde{\boldsymbol{w}}^{n}\right) \boldsymbol{u}^{n+1} \cdot \boldsymbol{v} d \boldsymbol{x}+\int_{\Omega^{n+1}} \frac{2}{\operatorname{Re}} \mathcal{E}\left(\boldsymbol{u}^{n+1}\right): \mathcal{E}(\boldsymbol{v}) d \boldsymbol{x} \\
\quad-\int_{\Omega^{n+1}} p^{n+1} \operatorname{div}(\boldsymbol{v}) d \boldsymbol{x}+\int_{\Omega^{n+1}} \frac{1}{2} \operatorname{div}\left(\tilde{\boldsymbol{u}}^{n}\right) \boldsymbol{u}^{n+1} \cdot \boldsymbol{v} d \boldsymbol{x} \\
=\frac{1}{\delta t} \int_{\Omega^{n}} \boldsymbol{u}^{n} \cdot\left(\boldsymbol{v} \circ \mathcal{A}_{n, n+1}\right) d \boldsymbol{x}+\int_{\Omega^{n+1}} \frac{1}{\mathrm{Fr}^{2}} \boldsymbol{\Theta} \cdot \boldsymbol{v} d \boldsymbol{x}, \\
\int_{\Omega^{n+1}} q \operatorname{div}\left(\boldsymbol{u}^{n+1}\right) d \boldsymbol{x}=0,
\end{array}\right.
$$

where $\tilde{\boldsymbol{u}}^{n}=\boldsymbol{u}^{n} \circ \mathcal{A}_{n, n+1}^{-1}$ and $\tilde{\boldsymbol{w}}^{n}=\boldsymbol{w}^{n} \circ \mathcal{A}_{n, n+1}^{-1}$. In practice, all these integrals are easy to evaluate since they involve functions defined at the same discrete time (either $t^{n}$ or $t^{n+1}$ ) and discretized on the same mesh (either $\mathcal{M}^{n}$ or $\mathcal{M}^{n+1}$ ), thereby avoiding any re-interpolation. Furthermore, the term $\int_{\Omega^{n+1}} \frac{1}{2} \operatorname{div}\left(\tilde{\boldsymbol{u}}^{n}\right) \boldsymbol{u}^{n+1} \cdot \boldsymbol{v} d \boldsymbol{x}$ is analogous to the well-known consistent modification introduced by Temam for the convective term to recover at the discrete level the skew-symmetry property of the advection term and ensure better stability properties. The resolution of the linear system (35) in $\left(\boldsymbol{u}^{n+1}, p^{n+1}\right)$ is performed by a GMRES iterative procedure with an ILU preconditioner and $\left(\boldsymbol{u}^{n}, p^{n}\right)$ as the initial guess. The most important computational task consists in building the matrix and the right-hand side, which change from one time step to another because of the moving mesh. Finally, we notice that even if the space discretization of the linearized Navier-Stokes system is implicit, the explicit computation of the domain velocity leads to a CFL-like restriction on the time step.

\subsection{Computing the domain velocity}

To complete the presentation of the numerical scheme, it remains to describe how the domain velocity $\boldsymbol{w}^{n}$ is computed matching the boundary conditions (4), (5), and (11). In addition, $\mathcal{A}_{n, n+1}$ defined from $\boldsymbol{w}^{n}$ by (32) must be sufficiently smooth so that the mesh remains regular enough for finite element computations. For the present problems, a simple method is to solve a Poisson problem. This approach can be seen as a simple device to extrapolate smoothly the mesh velocity from the boundaries to the whole domain. Moreover, we choose the mesh displacement to be along one direction only (along the coordinate axis associated with $x_{2}$ ), so that 
$\boldsymbol{w}^{n}=\left(0, w_{2}^{n}\right)$ and we solve the following scalar Poisson problem for $w_{2}^{n}$ :

$$
\begin{cases}-\Delta w_{2}^{n}=0 & \text { in } \Omega^{n}, \\ w_{2}^{n}=\frac{\boldsymbol{u}^{n} \cdot \boldsymbol{n}_{h}}{n_{h, 2}} & \text { on } \Sigma\left(t^{n}\right), \\ w_{2}^{n}=0 & \text { on } \Gamma_{\text {bot }}, \\ w_{2}^{n} \text { periodic } & \text { on } \Gamma_{\text {in/out }}\left(t^{n}\right) .\end{cases}
$$

This problem is discretized using the same finite element space as for the components of the discrete velocity $\boldsymbol{u}^{n}$. Moreover, the Dirichlet boundary condition on $\Sigma\left(t^{n}\right)$ is, as usual, enforced nodally, which requires to define an approximate normal vector $\boldsymbol{n}_{h}$ (with Cartesian components $\left(n_{h, 1}, n_{h, 2}\right)$ ) at each node of $\Sigma\left(t^{n}\right)$. The vector $\boldsymbol{n}_{h}$ can be defined in such a way that the Stokes formula holds true, thereby ensuring exact volume conservation at the discrete level; we refer to $[5, \S 5.1 .3 .2]$ for further details.

\subsection{Complete algorithm}

We can now write the complete algorithm. Suppose that $\Omega^{n}$ and $\left(\boldsymbol{u}^{n}, p^{n}\right)$ are known. Then $\boldsymbol{w}^{n}, \Omega^{n+1}$, and $\left(\boldsymbol{u}^{n+1}, p^{n+1}\right)$ are computed as follows:

(i) Compute the terms defined on $\Omega^{n}\left(\right.$ such as $\left.\frac{1}{\delta t} \int_{\Omega^{n}} \boldsymbol{u}^{n} \cdot\left(\boldsymbol{v} \circ \mathcal{A}_{n, n+1}\right) d \boldsymbol{x}\right)$ in the system (35).

(ii) Compute $\boldsymbol{w}^{n}=\left(0, w_{2}^{n}\right)$ by solving (36).

(iii) Move the nodes of the mesh according to $\mathcal{A}_{n, n+1}$ defined by (32).

(iv) Compute the remaining terms (defined on $\Omega^{n+1}$ ) in the system (35).

(v) Solve (35) to determine $\left(\boldsymbol{u}^{n+1}, p^{n+1}\right)$.

\section{Results}

In this section, we first present our numerical protocol to compute the critical Reynolds number for the onset of surface wave instabilities. Then, we discuss our results concerning the dependence of the critical Reynolds number on the inclination angle $\theta$, the waviness parameter $\zeta$, and the wavelength parameter $\xi$. A brief comparison with a shallow-water model is also presented at the end of the section.

We consider structured quadrangular meshes with typically 240 mesh cells in the $x_{1}$-direction and 30 cells in the $x_{2}$-direction. Typical nondimensional time steps $\delta t$ are in the interval $\left[10^{-3}, 5 \times 10^{-3}\right]$. We have 
verified the convergence of our numerical solutions by halving the mesh size and the time step in the most unfavorable cases for the Reynolds number (e.g., $\operatorname{Re}=90.9, \theta=\pi / 180, \zeta=0.033 \pi$, and $\xi=0.083 \pi$ ).

\subsection{Numerical protocol}

The numerical protocol to determine the critical Reynolds number consists of an extrapolation procedure relying on the time evolution of the free surface returning to equilibrium in stable flow configurations where the Reynolds number is close to, but smaller than, the critical threshold. First, a low enough Reynolds number (yielding a stable flow) is selected by choosing a large enough value for the viscosity, and a steady-state solution is computed. Then, this steady-state solution is used as an initial condition for a new calculation with a higher Reynolds number (lower viscosity). If the Reynolds number is still below the critical value, the solution will relax to a new steady-state. Consider a fixed observation point $x_{1} \in \mathbb{T}$. Then, referring to Figure 2, after a short transient of duration $T_{1}$, we observe that the film thickness at $x_{1}$, say $f(t):=h\left(t, x_{1}\right)$, exhibits the following behavior in time

$$
f(t) \simeq \varphi_{a, B, M, \omega}(t):=a \cos (\omega t) \exp (-B t)+M, \quad t \geq T_{1},
$$

for scalar positive real numbers $a, B, M$, and $\omega$ ( $a$ being the amplitude of the signal, $B$ the rate of decay, $M$ the mean value, and $\omega$ the frequency). The time $T_{1}$ can be taken as the time needed by the flow to cross two times the periodic domain, that is, $T_{1}=2 L / U_{*}$. Recalling the time scale $t_{*}=$ $h_{*} / U_{*}$, this yields in non-dimensional form $T_{1} / t_{*}=2 L / h_{*}=4 \pi / \xi$. In the present context, the most interesting coefficient appearing in (37) is $B$, which will be used to determine the critical Reynolds number by extrapolation as described below. We also observe from Figure 2 that the amplitude of oscillations is quite small, less than a percent. For larger Reynolds number, e.g., in the case $\theta=\frac{\pi}{180}$ where $\operatorname{Re} \sim 65$, the amplitude is of the order of a few percent.

A simple and cost-effective way to determine the coefficients $a, B$, and $M$ is to consider the local maxima of $f$ for $t \in\left[\lambda_{*} T_{1}, \lambda^{*} T_{1}\right]$, where $T_{1}$ is defined above, while $\lambda_{*}$ and $\lambda^{*}$ (with $1 \leq \lambda_{*}<\lambda^{*}$ ) are parameters defining the observation window in time (see below). This yields a series of pairs $\left\{\left(t_{i}, f\left(t_{i}\right)\right)\right\}_{1 \leq i \leq I}$ such that $f$ is locally maximal at $t_{i}$ (the corresponding points are indicated in Figure 2 for $\lambda_{*}=1$ and $\left.\lambda^{*}=6\right)$. Then, the coefficients $a, B$, and $M$ are obtained by minimizing the least-squares error

$$
\sum_{i=1}^{I}\left(f\left(t_{i}\right)-\bar{\varphi}_{a, B, M}\left(t_{i}\right)\right)^{2},
$$




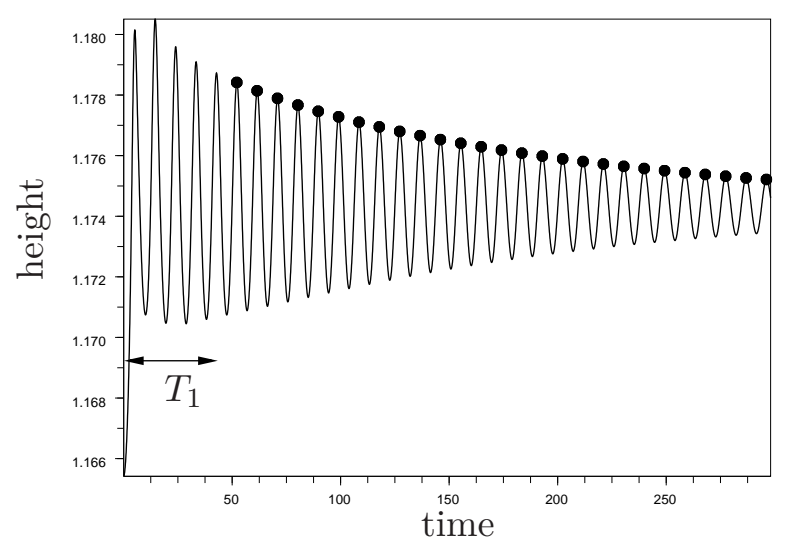

Figure 2: Example of relaxation back to equilibrium for a generic point on the free surface (corresponding to $x_{1}=0$ ) with $T_{1} \sim 48 t^{*} ; \operatorname{Re}=15.29, \theta=\frac{4 \pi}{180}, \zeta=0.016 \pi, \xi=0.083 \pi$.

where $\bar{\varphi}_{a, B, M}(t)=a \exp (-B t)+M$. The result is presented in Figure 3 for a typical flow configuration and shows excellent agreement. Finally, the coefficient $\omega$ can be obtained by a Fast Fourier Transform (FFT) of the function $(f(t)-M) \exp (B t) / a$. The result is shown in Figure 4 and is quite satisfactory: a marginal part of the energy of the Fourier transform is present at low frequency and corresponds to the initial transient behavior, while most of the energy concentrates around a single frequency; the second harmonic is also slightly visible.

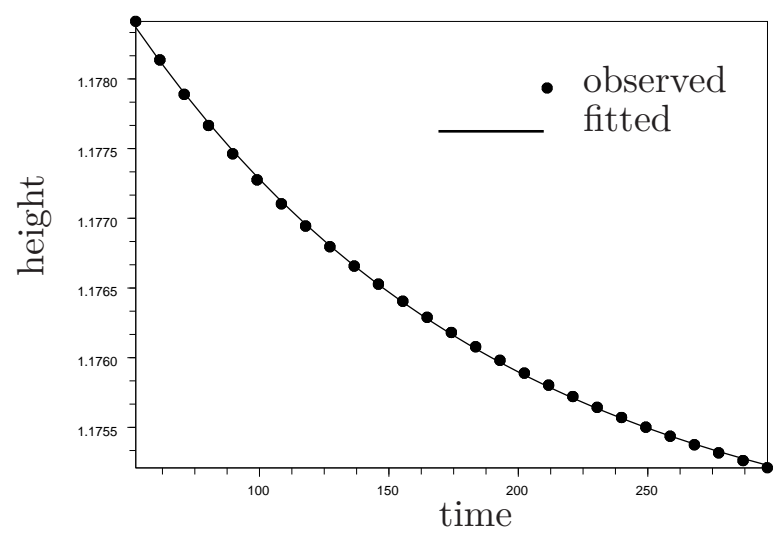

Figure 3: Fitting of the local maxima of $f$ : the bullets correspond to the points marked in Figure 2, and the solid line represents the function $\bar{\varphi}_{a, B, M}$ with the coefficients $a, B$, and $M$ determined from the least-squares fit.

The above numerical protocol can be used for all the points at the free 


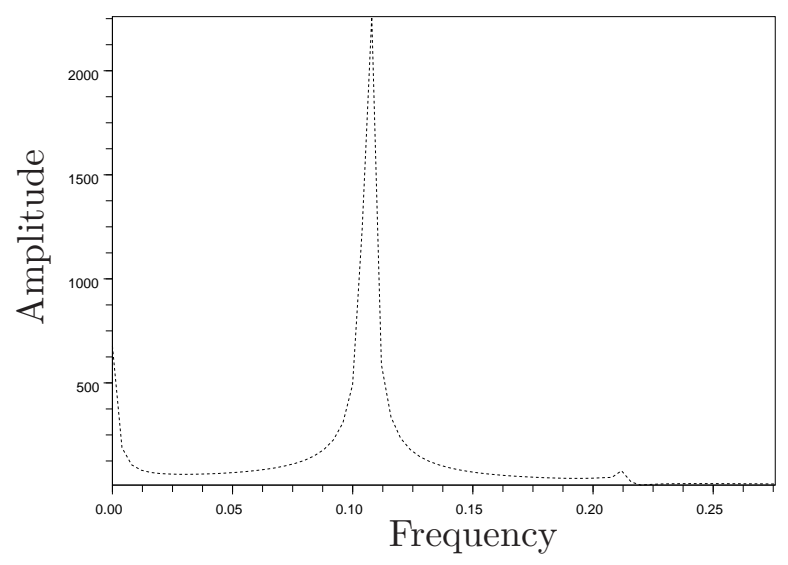

Figure 4: FFT of the function $(f(t)-M) \exp (B t) / a$.

surface. We have verified in all cases that the computed coefficients do not vary more than a few percent when another point $x_{1}$ is considered. In the numerical results reported below, we have used the mean value in space over all the mesh nodes on the free surface to evaluate the coefficient $B$. Two points are worth mentioning. First, the exponential decay regime takes longer to establish for higher Reynolds numbers, typically leading to observation windows with parameters up to $\lambda^{*}=2 \lambda_{*}=20$, while the choice $\lambda^{*}=2 \lambda_{*}=10$ is sufficient at moderate Reynolds numbers. Second, for the case of monotonously falling bottom considered in [13], relaxation times are shorter (typically $\lambda_{*}=2$ ), so that simulations are much less demanding. Finally, while we will mainly use the coefficient $B$ obtained from the above protocol, we observe that the coefficient $M$ is also useful since it provides the value for the film thickness at steady-state at the observation point $x_{1}$, without actually running the computations until steady-state (see, e.g., Figure 7 for an illustration). The flow rate at steady-state can also be obtained by applying the same procedure on the time-dependent flow rate.

Let $\eta_{1}$ and $\eta_{2}$ be two values of the viscosity yielding, respectively, the values $\mathrm{Re}_{1}$ and $\mathrm{Re}_{2}$ for the Reynolds number, and such that the flow is stable. By the above numerical protocol, we obtain relaxation coefficients $B_{1}$ and $B_{2}$, respectively. Then, we define the critical value of the Reynolds number, $\mathrm{Re}_{c}$, as the value for which the decay coefficient $B$ is zero. This value can be determined by linear extrapolation on the viscosity, or equivalently on $\operatorname{Re}^{-1 / 2}$ (see (26)), that is,

$$
\operatorname{Re}_{c}^{-1 / 2}=\frac{\operatorname{Re}_{1}^{-1 / 2} B_{2}-\operatorname{Re}_{2}^{-1 / 2} B_{1}}{B_{2}-B_{1}} .
$$


For the extrapolated value to be accurate, $\operatorname{Re}_{1}^{-1 / 2}$ and $\operatorname{Re}_{2}^{-1 / 2}$ need to be sufficiently close to the critical value $\operatorname{Re}_{c}^{-1 / 2}$. We have verified in all cases that these quantities departed by less than $5 \%$ from the computed value by extrapolation. A similar extrapolation procedure is used for the steady-state flow rate at the critical Reynolds number, which we denote by $Q_{c}$.

\subsection{Stability results}

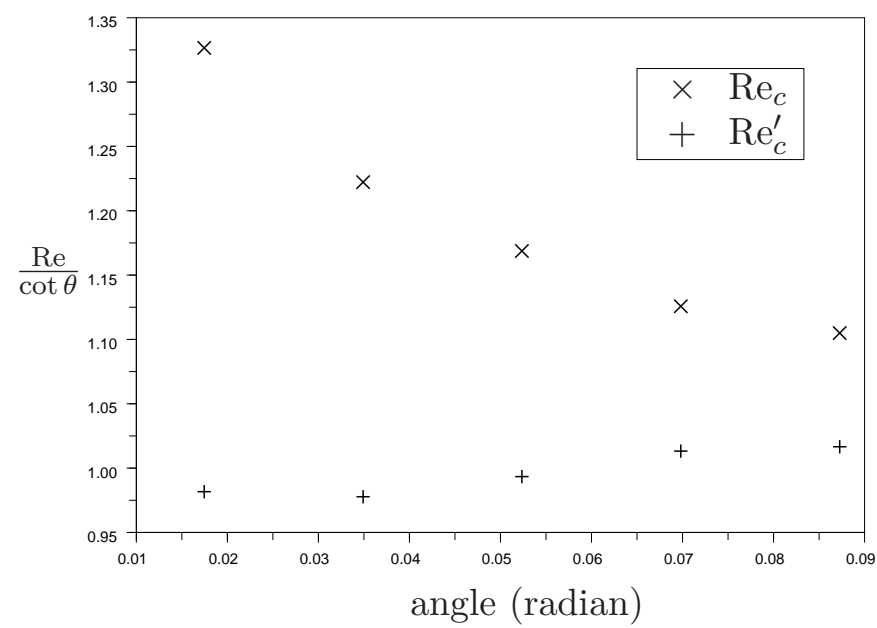

Figure 5: Critical Reynolds number versus inclination angle $\theta ; \zeta=0.016 \pi$ and $\xi=0.083 \pi$.

In this section, we study the dependence of the critical Reynolds number on the inclination angle $\theta$, the waviness parameter $\zeta$, and the wavelength parameter $\xi$. First, we fix $\zeta=0.016 \pi$ and $\xi=0.083 \pi$ (corresponding to $A / h_{*}=0.2$ and $\left.L / h_{*}=24\right)$ and let $\theta$ vary between $\frac{\pi}{180}$ and $\frac{5 \pi}{180}$. Figure 5 presents the results. We observe that the dependence of the critical Reynolds number on the inclination angle $\theta$ is not through $\cot \theta$ since the ratio $\operatorname{Re}_{c} / \cot \theta$ decreases with $\theta$. Since for Nusselt flow, this ratio is actually determined by the vertical average of the quadratic streamwise velocity, we can rescale the critical Reynolds number by using the extrapolated flow rate $Q_{c}$ instead of the Nusselt flow rate for flat incline $Q_{*}$; the resulting Reynolds number, denoted by $\operatorname{Re}_{c}^{\prime}$, is such that $\operatorname{Re}_{c}^{\prime}=\operatorname{Re}_{c}\left(Q_{c} / Q_{*}\right)$. As shown in Figure 5, the ratio $\operatorname{Re}_{c}^{\prime} / \cot \theta$ is now practically independent of $\cot \theta$. An interpretation of this result is that the velocity profile is still relatively close to parabolic and that the main modification caused by the topography waviness on the velocity profile is essentially through the flow rate. 


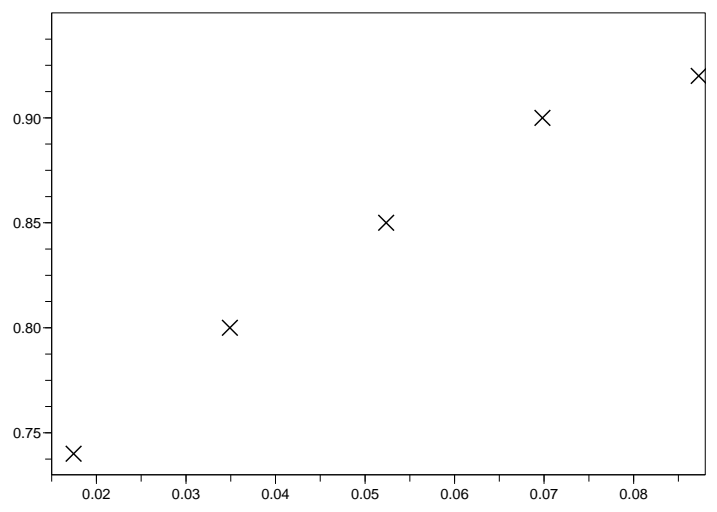

Figure 6: $Q_{c} / Q_{*}$ versus inclination angle $\theta ; \zeta=0.016 \pi$ and $\xi=0.083 \pi$.
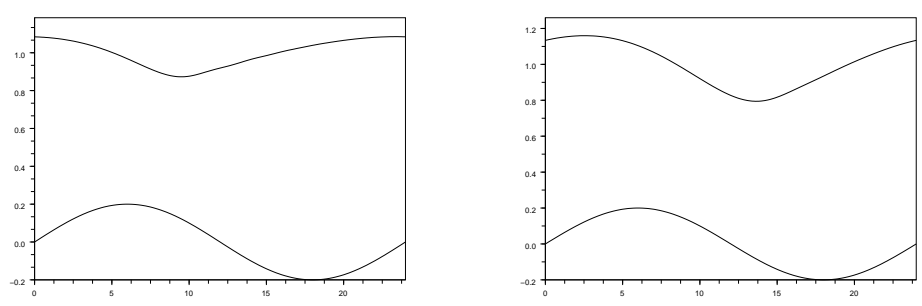

Figure 7: Free surface at steady-state for $\zeta=0.016 \pi$ and $\xi=0.083 \pi$; Left: $\operatorname{Re}=48$ and $\theta=1 \pi / 180 ;$ Right: $\operatorname{Re}=14.5$ and $\theta=4 \pi / 180$.

The ratio $Q_{c} / Q_{*}$ is presented in Figure 6 as a function of inclination angle, still for $\zeta=0.016 \pi$ and $\xi=0.083 \pi$. As expected, the waviness of the topography has a more pronounced effect on the flow rate at smaller inclination angles; our computations show that $Q_{c}$ is about $25 \%$ smaller than $Q_{*}$ for $\theta=\frac{\pi}{180}$. Furthermore, Figure 7 presents free surface profiles at steady-state for two inclination angles $\left(\theta=\frac{\pi}{180}\right.$ and $\left.\theta=\frac{4 \pi}{180}\right)$ and for a Reynolds number lower than, but close to, the critical value. We observe that the shapes of the two free surfaces are rather different. Indeed, although the corresponding inclination angles are fairly close, the Reynolds number for $\theta=\frac{\pi}{180}$ is larger, and the free surface height becomes minimal much closer to the hump in the topography.

The free-surface Navier-Stokes simulations allow one to explore criti- 


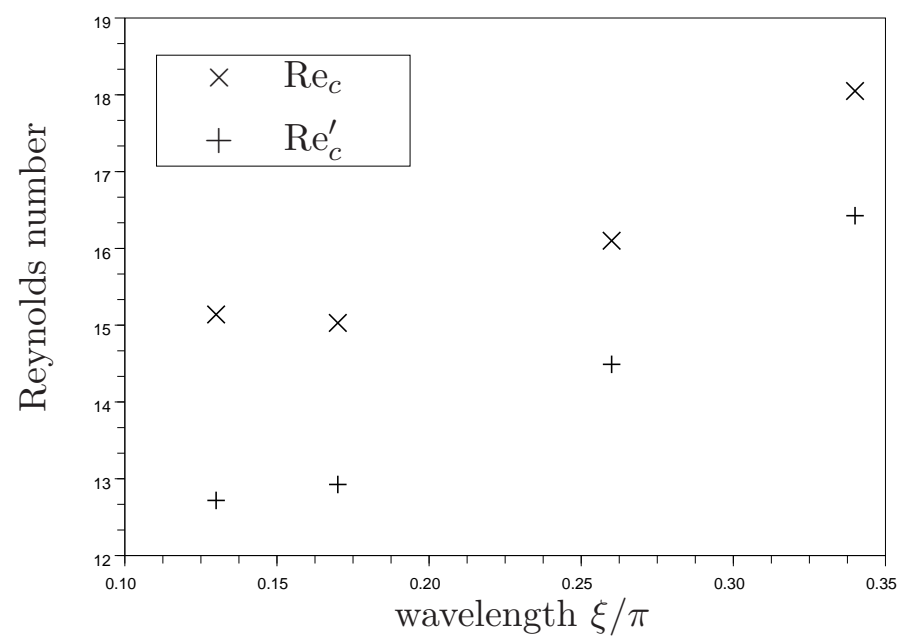

Figure 8: Critical Reynolds number as a function of wavelength parameter $\xi ; \theta=\frac{4 \pi}{180}$, $\zeta=0.016 \pi$.

cal Reynolds numbers for various values of the wavelength parameter $\xi$, whereas, in the classical theory of surface wave instability, the critical Reynolds number is estimated in the asymptotic limit $\xi \rightarrow 0$. To examine this point, we fix the inclination angle at $\theta=\frac{4 \pi}{180}$ and the waviness parameter at $\zeta=0.016 \pi$ and present in Figure 8 the critical Reynolds numbers $\operatorname{Re}_{c}$ and $\operatorname{Re}_{c}^{\prime}$ for $\xi / \pi \in\{0.111,0.083,0.055,0.042\}$ (corresponding to $\left.L / h_{*} \in\{18,24,36,48\}\right)$. As expected, the critical Reynolds number is an increasing (resp., decreasing) function of $\xi$ (resp., $L$ ). The results in Figure 8 indicate that the asymptotic regime $\xi \rightarrow 0$ is reached for the two smallest values for $\xi$. For the intermediate value $\xi=0.083 \pi$ considered in Figure 5 , the critical Reynolds number is about $6 \%$ larger than in the asymptotic regime $\xi \rightarrow 0$, and it is substantially larger for the highest value $\xi=0.111 \pi$. Figure 9 presents free surface profiles at steady-state for $\xi=0.11 \pi$ (left) and $\xi=0.055 \pi$ (right); compare also with the right panel of Figure 7 corresponding to the intermediate value $\xi=0.083 \pi$. The amplitude of bottom undulation is larger in the right panel than in the left since both settings correspond to the same value for the waviness parameter.

We now investigate the influence of the waviness parameter $\zeta$ on the critical Reynolds number. We fix the inclination angle at $\theta=\frac{4 \pi}{180}$ and the wavelength parameter at $\xi=0.083 \pi$. Critical Reynolds numbers are presented in Figure 10 for $\zeta / \pi \in\{0.017,0.021,0.025,0.029,0.033\}$ corresponding to $\left.A / h_{*} \in\{0.2,0.25,0.3,0.35,0.4\}\right)$. The critical Reynolds num- 

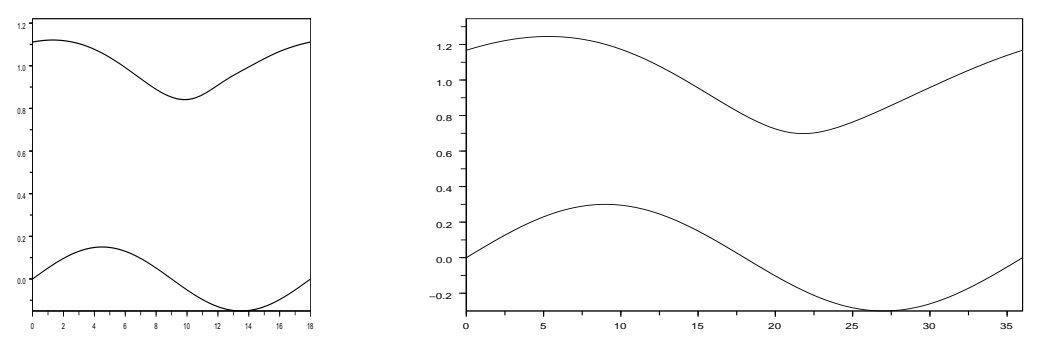

Figure 9: Free surface at steady-state for $\theta=4 \pi / 180$ and $\zeta=0.016 \pi$; Left: $\operatorname{Re}=15.2$ and $\xi=0.11 \pi$; Right: $\operatorname{Re}=12.57$ and $\xi=0.055 \pi$.

ber $\operatorname{Re}_{c}$ increases with the waviness parameter, indicating that the presence of waviness in the topography tends to stabilize the flow as in the case of monotonously falling bottom [13]. Interestingly, the Reynolds number $\operatorname{Re}_{c}^{\prime}$ evaluated with the critical flow rate is much less sensitive to $\zeta$. Thus, the main effect of waviness is lowering the actual flow rate, thereby tempering the previous conclusion on the stabilizing effect of waviness. The ratio $Q_{c} / Q_{*}$ decreases with $\zeta$ from about $90 \%$ for $\zeta=0.0166 \pi$ to about $65 \%$ for $\zeta=0.033 \pi$. Figure 11 presents free surface profiles at steady-state for the waviness parameter equal to $\zeta=0.025 \pi$ (left) and $\zeta=0.033 \pi$ (right); compare also with the right panel of Figure 7 corresponding to the lower value $\zeta=0.0166 \pi$. The impact of the waviness parameter on the shape of the steady-state free surface is clearly visible.

\begin{tabular}{|c|c|cc|c|}
\hline$\theta$ (deg) & $\zeta$ & \multicolumn{2}{|c|}{ free-surface Navier-Stokes } & {$[13]$} \\
& & $\xi=0.083 \pi$ & $\xi=0.042 \pi$ & $\xi \rightarrow 0$ \\
\hline 4 & 0.01 & 14.85 & 12.69 & 12.16 \\
4 & 0.02 & 14.97 & 13.01 & 12.99 \\
\hline 15 & 0.1 & 4.25 & 3.91 & 3.65 \\
\hline
\end{tabular}

Table 1: Critical Reynolds number computed using the present free-surface Navier-Stokes solver and the analytical result of [13] for various values of the inclination angle $\theta$ and the waviness parameter $\zeta$.

To assess our results, Table 1 compares the critical Reynolds number computed using the present free-surface Navier-Stokes solver and the analytical result of [13] for various values of the inclination angle $\theta$ and the waviness parameter $\zeta$. In [13], the condition of monotonously falling bottom restricts the value of the waviness parameter to $\zeta<0.07$ for $\theta=\frac{4 \pi}{180}$ and 


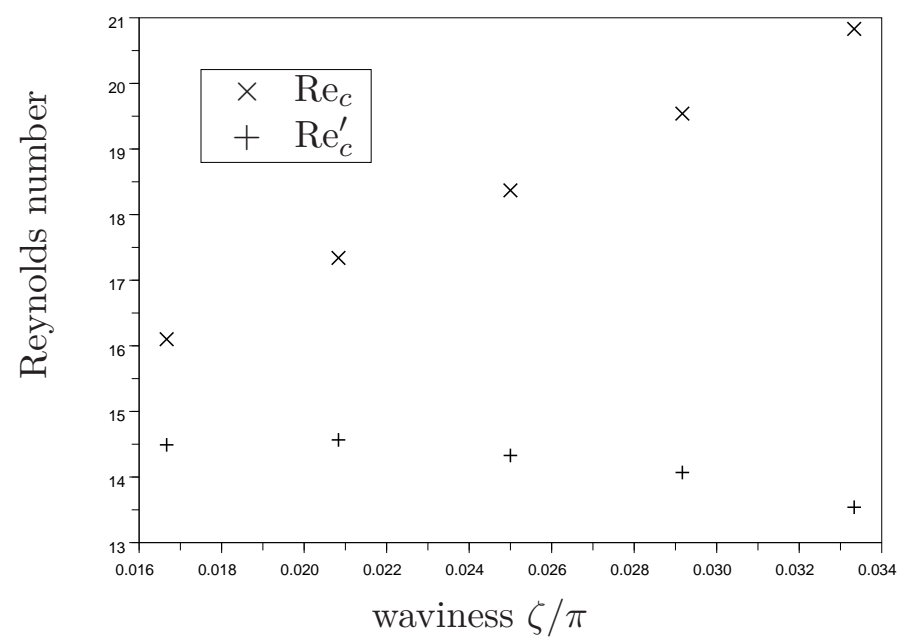

Figure 10: Critical Reynolds number as a function of waviness parameter $\zeta ; \theta=\frac{4 \pi}{180}$, $\xi=0.083 \pi$.

to $\zeta<0.27$ for $\theta=\frac{15 \pi}{180}$. First, we observe that both the present approach (with $\xi$ small enough) and that of [13] yield the same value for the critical Reynolds number as $\zeta \rightarrow 0$, namely the value $(5 / 6) \cot \theta$ corresponding to a flat incline (yielding $\operatorname{Re}_{c}=11.9$ for $\theta=\frac{4 \pi}{180}$ and $\operatorname{Re}_{c}=3.1$ for $\theta=\frac{15 \pi}{180}$ ). In the three configurations reported in Table 1, we observe that the computed critical Reynolds number with wavelength parameter $\xi=0.083 \pi$ is overestimated, while the value obtained for $\xi=0.042 \pi$ is reasonable close to that of [13]. A good agreement is achieved even for the waviness parameter $\zeta=0.1$ in the case $\theta=\frac{15 \pi}{180}$ where the Reynolds number is still small enough as assumed in [13]. As a further comparison, Figure 12 presents the free surface at steady-state obtained with the present approach and that of [13] for $\theta=15 \pi / 180$ and $\zeta=0.1$. For $\xi=0.083 \pi$, differences can be observed, while close agreement is obtained for $\xi=0.042 \pi$.

\subsection{Comparison with a shallow-water model}

For completeness, we present a brief comparison between the previous ALE Navier-Stokes results and those obtained with a steady shallow-water model. The latter is a simplified form drawn from a family of models derived by Boutonet, Chupin, Noble, and Vila [2] where we omit, in particular, surface tension and some high-order terms. The present steady shallowwater model is one-dimensional (primes denote derivatives with respect to 

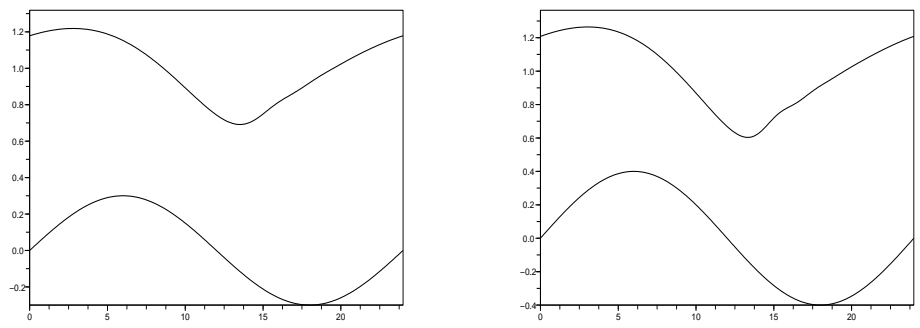

Figure 11: Free surface at steady-state for $\theta=4 \pi / 180$ and $\xi=0.083 \pi$; Left: $\operatorname{Re}=16.5$ and $\zeta=0.025 \pi ;$ Right: $\operatorname{Re}=19$ and $\zeta=0.033 \pi$.
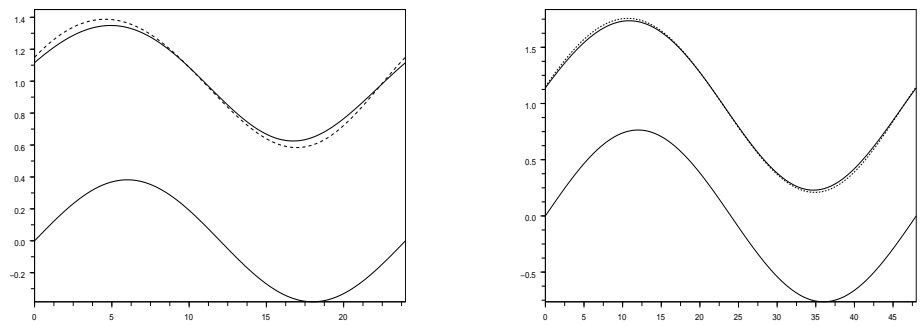

Figure 12: Comparison of free surface profiles for $\theta=15 \pi / 180, \zeta=0.1$, and $\operatorname{Re}=2.16$ : solid line for the present approach and dashed line for [13]; Left: $\xi=0.083 \pi$; Right: $\xi=0.042 \pi$ (different horizontal scales are used in both frames).

$\left.x_{1}\right)$ and expresses conservation of mass and momentum in the form

$$
\left\{\begin{aligned}
q_{\mathrm{sw}}^{\prime} & =0, \\
h_{\mathrm{sw}}^{\prime}\left(1-\frac{6}{5} \frac{q_{\mathrm{sw}}^{2}}{g h_{\mathrm{sw}}^{3} \cos \theta}\right) & =\tan \theta-b^{\prime}-3 \frac{\eta}{\rho} \frac{q_{\mathrm{sw}}}{g \cos \theta h_{\mathrm{sw}}^{3}},
\end{aligned}\right.
$$

where $q_{\mathrm{sw}}$ is the flow rate and $h_{\mathrm{sw}}$ the film thickness. The first equation classically implies that $q_{\mathrm{sw}}$ is constant. On the right-hand side of the second equation, we recall from (1) that $b^{\prime}\left(x_{1}\right)=\zeta \cos \left(2 \pi x_{1} / L\right)$, while the third term is a friction term obtained assuming a Nusselt vertical velocity profile (see [2]). Using as before the quantities $h_{*}$ and $h_{*} U_{*}$ for nondimensionalization, where $U_{*}$ is the Nusselt flow velocity defined by (24), the momentum balance equation becomes (the same notation is used for $q_{\mathrm{sw}}$ 
and $\left.h_{\mathrm{sw}}\right)$

$$
h_{\mathrm{sw}}^{\prime}\left(1-q_{\mathrm{sw}}^{2} \frac{2}{5} \frac{\tan \theta}{h_{\mathrm{Sw}}^{3}} \operatorname{Re}\right)=\tan \theta\left(1-\frac{q_{\mathrm{sw}}^{2}}{h_{\mathrm{sw}}^{3}}\right)-b^{\prime},
$$

where the Reynolds number Re, which enters this equation as a parameter, is again defined by (26). In the present setting, we additionally enforce $h_{\mathrm{sw}}$ to be periodic and have mean-value equal to 1 so as to fix the total volume of fluid as for the free-surface Navier-Stokes equations. Numerically, we solve for the constant $q_{\mathrm{sw}}$ and the function $h_{\mathrm{sw}}$ using an iterative procedure: given a value for $q_{\mathrm{sw}}$ and $h_{\mathrm{sw}}\left(L / h_{*}\right)$ (at the right boundary), equation (41) is first integrated backwards in $x_{1}$ using a fourth-order Runge-Kutta method with a spatial step equal to that used in the ALE Navier-Stokes calculations; then, periodicity and volume conservation are checked and if they are not satisfied, new values for $q_{\mathrm{sw}}$ and $h_{\mathrm{sw}}\left(L / h_{*}\right)$ are selected based on dichotomy. Since each step of the iterative procedure only requires solving a one-dimensional problem, the overall cost for computing $q_{\mathrm{sw}}$ and $h_{\mathrm{sw}}$ is much smaller than that incurred by the ALE Navier-Stokes solver. The values for the Reynolds number considered in the present comparison are small enough so that the factor $\left(1-q_{\mathrm{sw}}^{2} \frac{2}{5} \frac{\tan \theta}{h_{\mathrm{sw}}^{3}} \mathrm{Re}\right)$ on the left-hand side of (41) does not vanish, and the numerical integration of (41) is straightforward.

\begin{tabular}{|c|c|cc|c|}
\hline$\xi$ & Re & $q_{\text {ns }}$ & $q_{\mathrm{sw}}$ & error \\
\hline $0.055 \pi$ & 12.6 & 0.867 & 0.868 & $4.03 \times 10^{-4}$ \\
$0.083 \pi$ & 13.8 & 0.895 & 0.893 & $1.83 \times 10^{-3}$ \\
$0.11 \pi$ & 14.5 & 0.916 & 0.912 & $4.22 \times 10^{-3}$ \\
\hline
\end{tabular}

Table 2: Comparison of normalized flow rates obtained with the free-surface Navier-Stokes model and the shallow-water model.

Results are presented for $\theta=4 \pi / 180, \zeta=0.016 \pi$, and the three wavelength parameters considered previously. Table 4.3 compares the flow rate $q_{\mathrm{ns}}$ computed by the free-surface Navier-Stokes model with $q_{\mathrm{sw}}$. As expected, the error decreases as the wavelength parameter $\xi$ becomes smaller; quite interestingly, the agreement between both flow rates is extremely good even for the higher values of $\xi$. Finally, Figure 13 compares free surface profiles and shows very good agreement even for relatively large values of the wavelength parameter. 

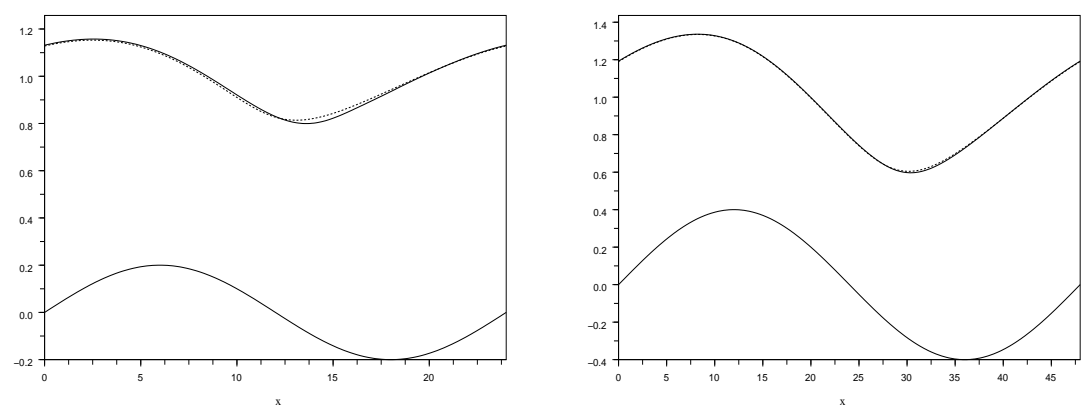

Figure 13: Comparison of free surface profiles for $\theta=4 \pi / 180$ and $\zeta=0.016 \pi$ : solid line for Navier-Stokes and dashed line for the shallow-water model; Left: Re $=13.8$ and $\xi=0.083 \pi$; Right: $\operatorname{Re}=12.01$ and $\xi=0.042 \pi$.

\section{Conclusions}

In this work, we have investigated numerically the stability of a thin liquid film flowing down an inclined wavy plane. We have used a direct numerical solver based on a finite element/ALE approximation of the freesurface Navier-Stokes equations. We have studied the dependence of the critical Reynolds number for the onset of surface wave instabilities on the inclination angle, the waviness parameter, and the wavelength parameter. We have focused on a specific parameter range with mild inclination owing to our targeted applications, but with relatively large waviness parameter so that the bottom can raise locally. We have obtained quantitative values for the critical Reynolds number using an extrapolation procedure based on the return to equilibrium of stable flows. In the present parameter range, higher amplitude and shorter wavelength for the bottom undulation stabilize the flow, the main effect of waviness being to lower the flow rate. The dependence of the critical Reynolds number evaluated with the Nusselt flow velocity on the inclination angle is more complex than through $\cot \theta$, but this dependence is recovered if the actual flow rate at critical conditions is used instead. Moreover, for small enough wavelength parameter, the present computations are in close agreement with the analytical results of [13] provided the waviness parameter is small enough for the bottom to fall monotonously. The present numerical approach to investigate flow stability still entails a substantial computational effort, especially to conduct systematic parametric studies. A typical runtime of the Navier-Stokes calculation on a workstation DELL Poweredge 1950 quadcore with $2 \times 2.50 \mathrm{GHz}$ cadenced processors to obtain a critical Reynolds number (comprising the calculation of two stable flows relaxing back to steady-state) ranges from 8 
hours to a couple of days depending on the time step, Reynolds number, and geometric parameters, the most demanding situation being $\xi \rightarrow 0$. An interesting perspective for the present study can be to carry out a classical spectral analysis using Floquet theory in the $x_{1}$ variable and based on the linearized Navier-Stokes equations around a computed steady-state.

Acknowledgments. This work was supported by the French National Research Agency (ANR) through the project METHODE \#ANR-07-BLAN0232. The authors wish to thank all the members of the project for fruitful discussions, and particularly Pablo Tassi, as well as J.-F. Gerbeau (INRIA).

\section{References}

[1] T. B. Benjamin. Wave formation in laminar flow down an inclined plane. J. Fluid Mechanics, 2:554-574, 1957.

[2] M. Boutonet, L. Chupin, P. Noble, and J.P Vila. Shallow water flows for arbitrary topography. Comm in Math Sciences, 6:29-55, 2008.

[3] J.-F. Gerbeau and T. Lelièvre. Generalized Navier boundary condition and geometric conservation law for surface tension. Computer Methods Appl. Mech. Engrg., 198(5-8):644-656, 2009.

[4] J.-F. Gerbeau, T. Lelièvre, and C. Le Bris. Simulations of MHD flows with moving interfaces. J. Comput. Phys., 184(1):163-191, 2003.

[5] J.-F. Gerbeau, T. Lelièvre, and C. Le Bris. Mathematical Methods for the Magnetohydrodynamics of Liquid Metals. Numerical Mathematics and Scientific Computation. Oxford University Press, 2006.

[6] J. Liu and J. P Gollub. Solitary wave dynamics of film flows. Phys. Fluids, 6:1702-1712, 1994.

[7] METHODE. Modélisation de l'écoulement sur une topographie avec des hétérogénéités orientées et différences d'échelles. http://methode.netcipia.net.

[8] W. Nusselt. Die Oberflachenkondensation des Wasserdampfes. Z. Ver. Dt. Ing., 60:541-552, 1916.

[9] B. Ramaswamy, S. Chippada, and S. W. Joo. A full-scale numerical study of interfacial instabilities in thin-film flows. J. Fluid Mechanics, 325:163-194, 1996. 
[10] C. Ruyer-Quil and P. Manneville. Modeling film flows down inclined planes. Eur. Phys. J. B, 6:277-292, 1998.

[11] V. Souchère, D. King, J. Darousin, F. Papy, and A. Capillon. Effects of tillage on runoff directions: consequences on runoff contributing areas within agricultural catchments. J. Hydrol., 206:256-267, 1998.

[12] R. L. Webb. Principles of Enhanced Heat Transfer. Wiley, New York, 1994.

[13] A. Wierschem and N. Aksel. Instability of a liquid film flowing down an inclined wavy plane. Physica D, 186:221-237, 2003.

[14] A. Wierschem, M. Scholle, and N. Aksel. Comparison of different theoretical approaches to experiments on film flow down an inclined wavy channel. Exper. Fluids, 33:429-442, 2002.

[15] C. S. Yih. Stability of liquid flow down an inclined plane. Phys. Fluids, 6(3):321-334, 1963. 